\title{
Measurement of Jet Production Cross Sections in Deep-inelastic ep Scattering at HERA
}

\author{
Daniel Britzger* for the H1 Collaboration \\ DESY, Notkestr. 85, 22607 Hamburg, Germany \\ E-mail: daniel.britzger@desy.de
}

A precision measurement of jet cross sections in neutral current deep-inelastic scattering for photon virtualities $5.5<Q^{2}<80 \mathrm{GeV}^{2}$ and inelasticities $0.2<y<0.6$ is presented, using data taken with the $\mathrm{H} 1$ detector at HERA, corresponding to an integrated luminosity of $290 \mathrm{pb}^{-1}$. Double-differential inclusive jet, dijet and trijet cross sections are measured simultaneously and are presented as a function of jet transverse momentum observables and as a function of $Q^{2}$. Jet cross sections normalised to the inclusive neutral current DIS cross section in the respective $Q^{2}$ interval are also determined. Previous results of inclusive jet cross sections in the range $150<Q^{2}<15000 \mathrm{GeV}^{2}$ are extended to low transverse jet jet momenta $5<P_{\mathrm{T}}<7 \mathrm{GeV}$. The data are compared to predictions from perturbative QCD in next-to-leading order in the strong coupling, in approximate next-to-next-to-leading order and in full next-to-next-to-leading order. Using also the recently published $\mathrm{H} 1$ jet data at high values of $Q^{2}$, the strong coupling constant $\alpha_{s}\left(m_{Z}\right)$ is determined in next-to-leading order to value of $\alpha_{s}\left(m_{Z}\right)=0.1172(4)_{\exp }\left({ }_{-45}^{+53}\right)_{\mathrm{th}}$.

XXV International Workshop on Deep Inelastic Scattering and Related Topics

3-7 April, 2017

University of Birmingham, $U K$

\footnotetext{
*Speaker.
} 


\section{Introduction}

The production of jets in neutral-current deep-inelastic electron-proton scattering (NC DIS) is an important process to study the phenomenology of QCD. The measurements of cross sections for jet producions are performed in the Breit frame, which is defined such that the incoming parton collides head-on with the exchanged virtual boson. Therefore, jet cross sections are sensitive to the strong coupling constant, $\alpha_{s}$, already in leading order, and are directly sensitive to the gluon content of the proton. The measurement presented here [U] complements an earlier measurement of jet cross sections by $\mathrm{H} 1$ [ []] to lower values of $Q^{2}$ and also extents its reach to lower values of $P_{\mathrm{T}}^{\mathrm{jet}}$. Predictions for jet production are obtained in the framework of perturbative $\mathrm{QCD}$, where the perturbatively calculated partonic cross section is convoluted with parton density functions of the proton (PDFs). These predictions are corrected for non-perturbative hadronisation effects obtained from Monte Carlo event generators (MC). The standard for the calculations of the partonic cross sections has been next-to-leading order (NLO) for a considerable time, but very recently nextto-next-to-leading order (NNLO) predictions were performed for the first time [ [3], 田]. Here, a first comparison of NNLO predictions to inclusive jet cross sections is presented, and the data-to-theory agreement is quantitatively investigated.

\section{Measurement of jet cross sections}

The measurement is based on data collected during the HERA-II running period in the years 2005 and 2007 and correspond to an integrated luminosity of $290 \mathrm{pb}^{-1}$. Jets are constructed in the Breit frame using the $k_{\mathrm{T}}$ jet-algorithm with a clustering parameter $R=1$.
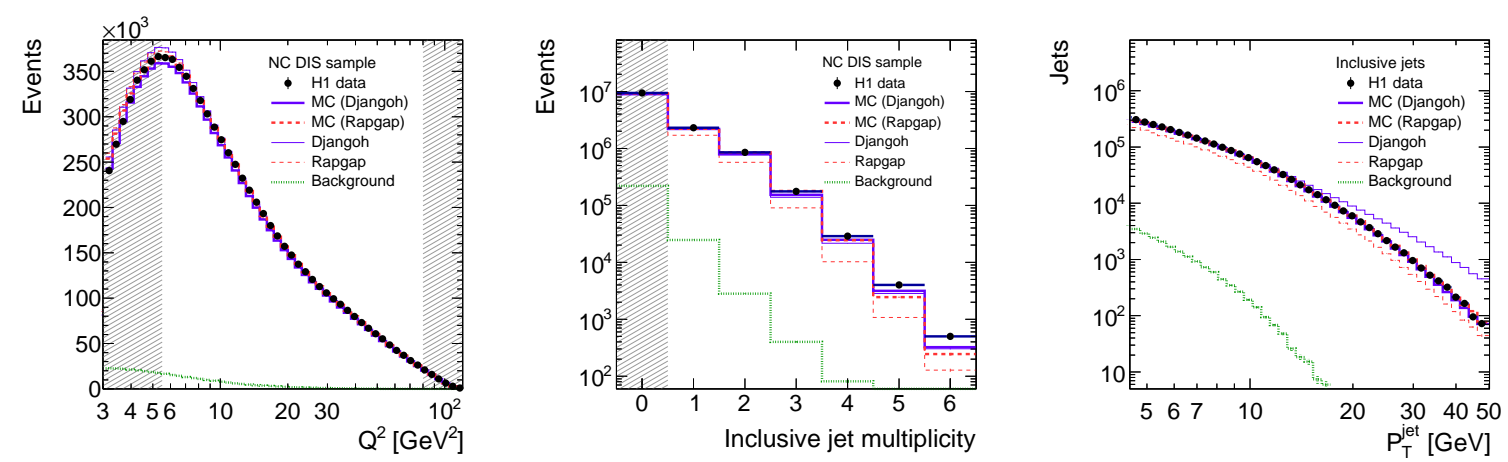

Figure 1: Comparison on detector level of data to MC predictions obtained from the Djangoh and Rapgap MC programs. From left to right: distributions of $Q^{2}$ for the selected neutral current DIS data on detector level, distributions of the multiplicy of jets in NC DIS events and distributions of the transverse momenta $P_{\mathrm{T}}^{\mathrm{jet}}$ of the inclusive jet measurement on detector level. The MC events (full line) are weighted to achieve a better description of the data, whereas the unweighted MC predictions (dashed lines) have partially problems describing the data.

In order to correct the data for detector effects due to limited resolution and acceptance, a regularised unfolding algorithm is employed [5]. The migration matrix, which is input to the TUnfold algorithm is constructed as a block matrix, where the submatrices represent the migrations of the inclusive neutral current DIS (NC DIS), inclusive jet, dijet and trijet cross sections. Further 
submatrices represent jets, or events, which have no counterpart on truth level, but their NC DIS kinematics are known, and thus they can be constraint by NC DIS events. Its entries are obtained from MC events, which are generated with the Djangoh [目] and Rapgap [U] MC event generator and weighted to data. In order to control migrations at the boundaries of the phase space, all kinematic requirements are relaxed for the unfolding compared to the phase space of the cross sections. For instance, the NC DIS events are selected in the kinematic range $3<Q^{2}<120 \mathrm{GeV}^{2}$ and jets must exceed a transverse momentum of $P_{\mathrm{T}}^{\mathrm{jet}}>3 \mathrm{GeV}$, whereas the measurement phase space is $5.5<Q^{2}<80 \mathrm{GeV}^{2}$ and jets are required to be $P_{\mathrm{T}}^{\mathrm{jet}}>4.5 \mathrm{GeV}$. Background contributions are generally modelled and are subtracted from data based on simulated photoproduction events which are normalised to a dedicated data selection.

Inclusive jet cross sections are then measured as a function of $Q^{2}$ and $P_{\mathrm{T}}^{\mathrm{jet}}$ for jets within the kinematic range $4.5<P_{\mathrm{T}}^{\text {jet }}<50 \mathrm{GeV}$ and in pseudorapidity of $-1.0<\eta_{\mathrm{lab}}^{\text {jet }}<2.5$. Cross sections for dijet and trijet production are defined by considering events with at least two (three) jets with $P_{\mathrm{T}}^{\mathrm{jet}}>4 \mathrm{GeV}$ within the pseudorapdity range $-1.0<\eta_{\mathrm{lab}}^{\mathrm{jet}}<2.5$ and are measured as a function of $Q^{2}$ and the average $P_{\mathrm{T}}^{\text {jet }}$ of the two or three leading jets, $\left\langle P_{\mathrm{T}}\right\rangle_{2}$ or $\left\langle P_{\mathrm{T}}\right\rangle_{3}$, respectively. The lower kinematic bounds of $\left\langle P_{\mathrm{T}}\right\rangle>5 \mathrm{GeV}$ and $\left\langle P_{\mathrm{T}}\right\rangle_{3}>5.5 \mathrm{GeV}$ taken together with the minimum $P_{\mathrm{T}}^{\text {jet }}$ of an individual jet of $4 \mathrm{GeV}$, ensure that regions are excluded, where the pQCD calculations are infared sensitive and thus are less reliable. Normalised jet cross sections are obtained by dividing by the measured inclusive NC DIS cross section in the respective $Q^{2}$ range.

The improved understanding of the low- $P_{\mathrm{T}}^{\text {jet }}$ region enabled to extent the phase space of a previous inclusive jet analysis at high- $Q^{2}\left[[]\right.$ to lower values of $P_{\mathrm{T}}^{\mathrm{jet}}$. This kinematic region was already included in the unfolding algorithm of that analysis, and thus these cross section measurements for $5<P_{\mathrm{T}}^{\mathrm{jet}}<7 \mathrm{GeV}$ as a function of $Q^{2}$ in the region $150<Q^{2}<15000 \mathrm{GeV}^{2}$ could now be provided as an extension of the kinematic reach.

\section{Results}

\subsection{Inclusive jet cross sections}

The cross sections for inclusive jet production as a function of $Q^{2}$ and $P_{\mathrm{T}}^{\mathrm{jet}}[\mathrm{W}]$ are presented in figure $\square$ and compared to predictions in NLO, in approximate NNLO using the threshold resummation formalism (aNNLO) [ [] and in full NNLO. The pQCD calculations employ the NNPDF3.0 parameterisation of the PDFs $[Q]$ and are corrected for hadronisation effects with factors determined from MC generators [6, $[$ ]].

The NLO predictions provide a reasonable description of the data over the full kinematic range within the experimental and theoretical uncertainties. The aNNLO predictions provide an improved description of the shape, but they have problems in the normalisation. The NNLO predictions provide a good description of the shape and the normalisation of the data in all kinematic regions. The NNLO calculations have reduced scale uncertianties than the NLO predictions, which is most significant for higher values of $Q^{2}$ or $P_{\mathrm{T}}^{\text {jet }}$. At lower scales, the precision of the data is significantly higher than the one of the NNLO predictions, while at higher scales it is opposite.

The inclusive jet cross sections are divided by the measured inclusive NC DIS cross section in the respective $Q^{2}$ bin, in order to obtain normalised inclusive jet cross sections. These are 

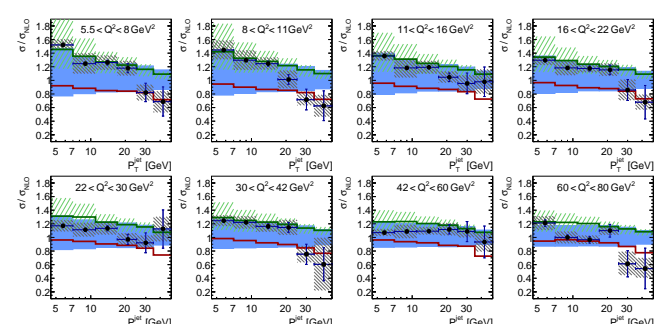

$\mathrm{H} 1$ Inclusive jets

$\nmid$ H1 HERA-II

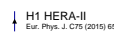
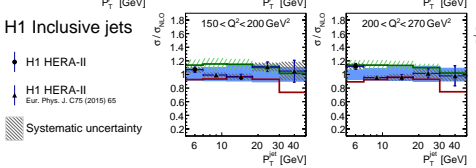

NLO $\otimes$ hadr. corr.

WN NNLO $\otimes$ hadr. corr.

- aNNLO $\otimes$ hadr. corr.
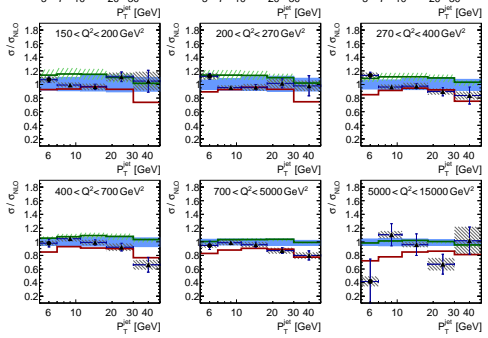

Figure 2: Ratio of inclusive jet cross sections to the NLO predictions and ratio of aNNLO and NNLO to NLO predictions as function of $Q^{2}$ and $P_{\mathrm{T}}^{\mathrm{jet}}$. Measurements at higher values of $Q^{2}$ and $P_{\mathrm{T}}^{\mathrm{jet}}>7 \mathrm{GeV}$ are taken from Ref. [D] and compared for the first time to NNLO predictions here.
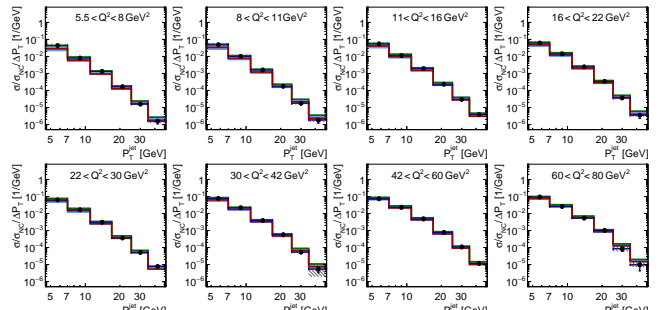

H1 Normalised

inclusive jets

4. H1 HERA-II

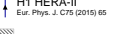

\begin{tabular}{llll} 
& \\
\hline
\end{tabular}
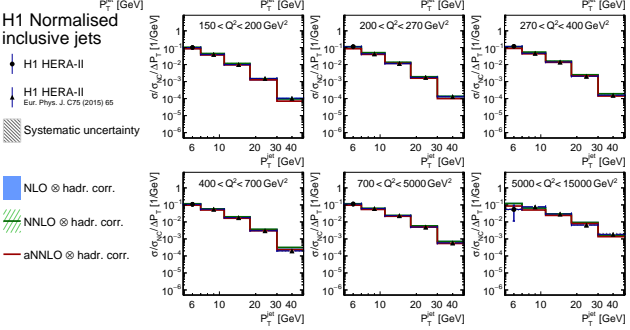

Figure 3: Normalised inclusive jet cross sections compared to NLO, aNNLO and NNLO predictions as a function of $Q^{2}$ and $P_{\mathrm{T}}^{\mathrm{jet}}$. The cross sections are divided in each bin by the bin size in $P_{\mathrm{T}}^{\text {jet }}$.

displayed together with theoretical predictions in figure [3. The normalised jet cross sections feature a reduction of the systematic uncertainties in comparison to non-normalised cross sections, because systematic uncertainties have been canceled partially. The normalised jet cross sections increase as a function of $Q^{2}$ for a given interval in $P_{\mathrm{T}}^{\mathrm{jet}}$. The NNLO predictions provide a good description of the normalised inclusive jet cross sections over the entire kinematic range.
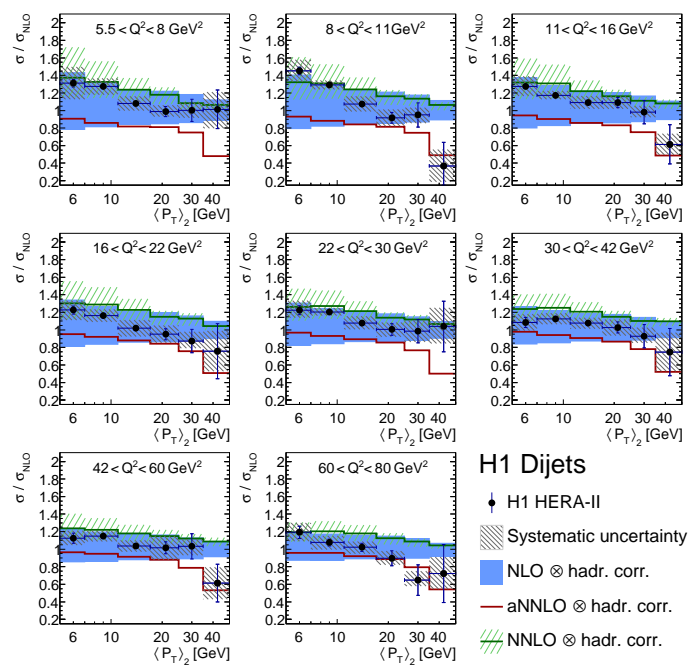

H1 Dijets

† H1 HERA-I

$\mathbb{N}$ Systematic uncertainty

$\mathrm{NLO} \otimes$ hadr. corr.

- aNNLO $\otimes$ hadr. corr.

HNLO $\otimes$ hadr. corr.

Figure 4: Ratio of dijet cross sections to NLO predictions and ratio of the aNNLO and NNLO predictions to the NLO predictions as a function of $Q^{2}$ and $\left\langle P_{\mathrm{T}}\right\rangle_{2}$.
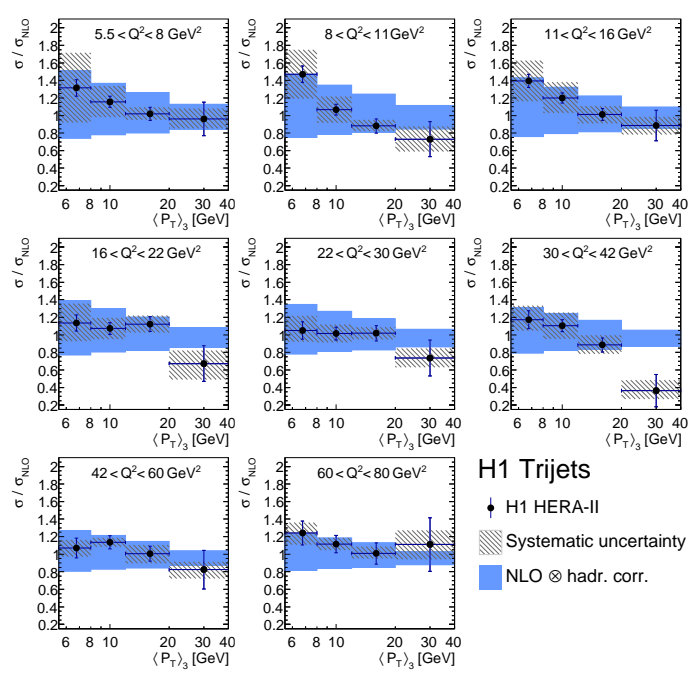

$\mathrm{H} 1$ Trijets

- H1 HERA-II

$\mathbb{N}$ Systematic uncertainty

NLO $\otimes$ hadr. corr.

Figure 5: Ratio of trijet cross sections to NLO predictions and as a function of $Q^{2}$ and $\left\langle P_{\mathrm{T}}\right\rangle_{3}$. 


\subsection{Dijet and trijet cross sections}

The ratio of double-differential dijet cross sections as function of $\left\langle P_{\mathrm{T}}\right\rangle_{2}$ and $Q^{2}$ together with the aNNLO and NNLO predictions to the NLO predictions are presented in figure $\rightarrow$. Within the scale uncertainties, the data are described well by the NLO predictions. The NNLO predictions provide a better description of the dijet cross section. This observation is also confirmed by the improved value of $\chi^{2} / n_{\text {dof }}$, which is 1.6 for NLO, and 1.0 for the NNLO dijet predictions.

The ratio of the double-differential trijet cross sections as function of $\left\langle P_{\mathrm{T}}\right\rangle_{3}$ and $Q^{2}$ to the NLO predictions is displayed in figure 5 . No aNNLO or NNLO predictions are yet computed for trijet cross sections. The NLO calculations provide an overall good description of thd data and the experimental uncertainty is typically smaller than the scale uncertainty of the NLO predictions. A trend to undershoot the data at lower values of $\left\langle P_{\mathrm{T}}\right\rangle_{3}$ is observed, which may indicate that higher orders are mandatory and that kinematic region.

Furthermore, normalised dijet and trijet cross sections have been measured and compared to the predictions and the features observed with the non-normalised cross sections are confirmed.

\subsection{Determination of the strong coupling constant}

The value of the strong coupling constant $\alpha_{s}\left(m_{Z}\right)$ is determined in a fit of NLO predictions to normalised inclusive jet, normalised dijet and normalised trijet cross sections. Data at lower values of $Q^{2}$, as presented in this analysis, and at high$Q^{2}$ from a previous analysis [] $]$ are considered. The value of $\alpha_{s}\left(m_{Z}\right)$ is determined to

$$
\begin{array}{r}
\alpha_{s}\left(m_{Z}\right)=0.1172(4)_{\exp }(3)_{\mathrm{PDF}}(7)_{\mathrm{PDF}\left(\alpha_{\mathrm{s}}\right)} \\
(11)_{\mathrm{PDFset}}(6)_{\text {had }}\left({ }_{-43}^{+51}\right)_{\text {scale }} .
\end{array}
$$

The fit yields a value of $\chi^{2} / n_{\mathrm{dof}}=1.36$ for 198 data points, whereas other PDF sets result in a smaller value of around 1.1. The uncertainties studied inlcude the experimental uncertainty, PDF uncertainties, uncertainties due to the assumption made on $\alpha_{s}$ for the PDF determination, uncertainties due to the choice of the PDF set, hadronisation and scale uncertainites. The largest uncertainty by far is the scale uncertainty of the NLO predictions, which is expected to be reduced once

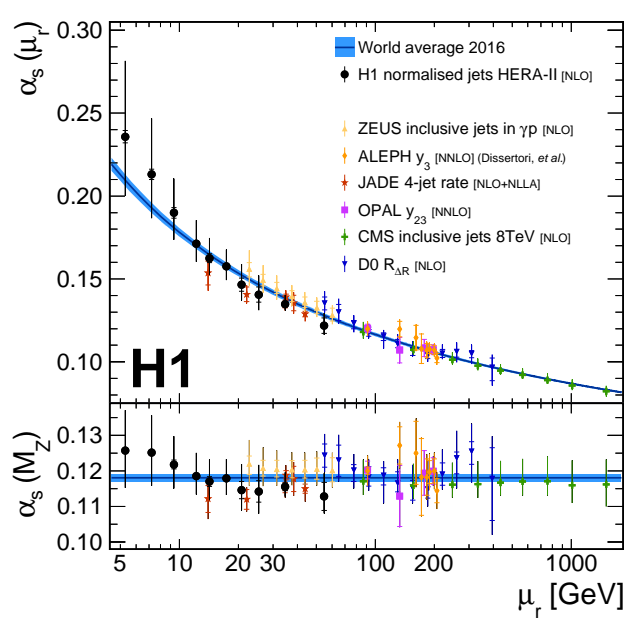

Figure 6: Values of $\alpha_{s}\left(m_{Z}\right)$ extracted from the normalised inclusive jet, dijet and trijet cross sections using NLO predictions compared to values extracted from other jetdata. The upper panel shows the values of the strong coupling $\alpha_{s}\left(\mu_{R}\right)$ and the lower panel the equivalent values of $\alpha_{s}\left(m_{Z}\right)$ for all measurements. the NNLO calculations are available for such fits.

In order to explore the running of the strong coupling, $\alpha_{s}\left(\mu_{R}\right)$, the data points are grouped into ten groups with comparable values for $\mu_{R}$, and the value of $\alpha_{s}\left(m_{Z}\right)$ is obtained from fits to each group separately. The results are compared to other determinations using jet data in figure 6 . 
The $\mathrm{H} 1$ jets data probes the running in the range of $5<\mu_{R}<90 \mathrm{GeV}$ and consistency with other extractions and with the world average value [ㅁ] is observed.

\section{Summary and conclusion}

Measurements of the inclusive jet, dijet and trijet cross sections in neutral current deep-inelastic $e p$ scattering in the range $5.5<Q^{2}<80 \mathrm{GeV}^{2}$, as well as these jet cross sections normalised to the inclusive NC DIS cross sections are presented. At values of $150<Q^{2}<15000 \mathrm{GeV}^{2}$ new cross section measurements for inclusive jet cross sections for jet transverse momenta of $5<P_{\mathrm{T}}^{\text {jet }}<7 \mathrm{GeV}$ are presented, extending the kinematic reach of already published results. New predictions in full next-to-next-to-leading order in perturbative QCD improve the descriptions for inclusive jet and dijet cross sections compared to NLO predictions, and give an overall good description of the data. The strong coupling $\alpha_{s}\left(m_{Z}\right)$ is determined in a fit of NLO predictions to the normalised inclusive jet, dijet and trijet cross sections in the range of $5.5<Q^{2}<15000 \mathrm{GeV}^{2}$ to a value of $\alpha_{s}\left(m_{Z}\right)=$ $0.1172(4)_{\exp }\left({ }_{-45}^{+53}\right)_{\mathrm{th}}$. The value is found to be consistent with other extractions and demonstrates the high experimental precision of the data.

\section{Acknowledgements}

We are thankful to T. Gehrmann, J. Niehues and G. Kramer for providing their predictions and for helpful discussions.

\section{References}

[1] H1 Collaboration, V. Andreev et al., Eur. Phys. J. C77 (2017) 215, arxiv:1611.03421.

[2] H1 Collaboration, V. Andreev et al. Eur. Phys. J. C75 (2015) 65, arX1v:1406.4709.

[3] J. Currie, T. Gehrmann, and J. Niehues, Phys. Rev. Lett. 117 (2016) 042001, arXiv: 1606.03991.

[4] J. Currie, T. Gehrmann, A. Huss, and J. Niehues, arXiv:1703.05977.

[5] S. Schmitt, JINST 7 (2012) T10003, arXiv:1205.6201.

[6] K. Charchula, G.A. Schuler and H. Spiesberger, Comp. Phys. Commun. 81 (1994) 381, DJANGOH V1.4

[7] H. Jung, Comp. Phys. Commun. 86 (1995) 147, RAPGAP V3.1

[8] T. Biekötter, M. Klasen, and G. Kramer Phys. Rev. D92 no. 7, (2015) 074037, arXiv:1508.07153.

[9] NNPDF Collaboration, R. D. Ball et al., VHEP 04 (2015) 040, arXiv: 1410.8849.

[10] Particle Data Group Collaboration, C. Patrignani et al., Chin. Phys. C40 (2016) 100001. 